[2] A. Isidori, Nonlinear Control Systems II. London, U.K.: Springer-Verlag, 1999.

[3] M. Krstić, D. Fontaine, P. V. Kokotovic, and J. D. Paduano, "Useful nonlinearities and global stabilization of bifurcations in a model of jet engine surge and stall," IEEE Trans. Automat. Contr., vol. 43, pp. 1739-1745, Dec. 1998.

[4] M. Krstić, I. Kanellakopoulos, and P. Kokotović, Nonlinear and Adaptive Control Design. New York: Wiley, 1995.

[5] D. Liaw and E. Abed, "Stability analysis and control of rotating stall," in IFAC Nonlinear Control Systems Design Symp., Bordeaux, France, June 1992.

[6] Output feedback control of non-UCO systems: The jet engine stall and surge example, M. Maggiore and K. M. Passino. (2002). http://www.control.utoronto.ca/tech_reports/rept0202.pdf [Online]

[7] - "A separation principle for a class of non-UCO systems," IEEE Trans. Automat. Contr., vol. 48, pp. 1122-1133, July 2003.

[8] F. K. Moore and E. M. Greitzer, "A theory of post-stall transients in axial compression systems-part I: development of equations," J. Turbomach., vol. 108, pp. 68-76, 1986.

[9] J. D. Paduano, L. Valavani, A. Epstein, E. Greitzer, and G. R. Guenette, "Modeling for control of rotating stall," Automatica, vol. 30, no. 9, pp. 1357-1373, Sept. 1994.

[10] A. Teel and L. Praly, "Global stabilizability and observability imply semi-global stabilizability by output feedback," Syst. Control Lett., vol. 22, pp. 313-325, 1994.

\section{Contractibility of Dynamic LTI Controllers Using Complementary Matrices}

Lubomír Bakule, José Rodellar, and Josep M. Rossell

\begin{abstract}
A generalized structure of complementary matrices involved in the input-state-output inclusion principle for linear time-invariant systems including contractibility conditions for static state feedback controllers is well known. In this note, it is shown how to further extend this structure when considering contractibility of dynamic controllers. Necessary and sufficient conditions for contractibility are proved in terms of both unstructured and block structured complementary matrices for general expansion/contraction transformation matrices. Explicit sufficient conditions for blocks of complementary matrices ensuring contractibility are proved for general expansion/contraction transformation matrices. Moreover, these conditions are further specialized for a particular class of transformation matrices.
\end{abstract}

Index Terms-Contractibility, decentralized control, dynamic controllers, estimators, inclusion principle, overlapping.

\section{INTRODUCTION}

The inclusion principle proposed in the context of analysis and control of complex and large scale systems in [10], [13], [14], and [16] establishes essentially a mathematical framework for two dynamic sys-

Manuscript received July 30, 2002; revised February 24, 2003. Recommended by Associate Editor A. Garulli. This work was supported in part by the Generalitat of Catalonia under Grant DURSI PIV, by the Academy of Sciences of the Czech Republic under Grant A2075802, and by the Committee for Science and Technology (CICYT) of Spain under Grant DPI2002-04018-C02-01.

L. Bakule is with the Institute of Information Theory and Automation, Academy of Sciences of the Czech Republic, 18208 Prague 8, Czech Republic (bakule@utia.cas.cz).

J. Rodellar and J. M. Rossell are with the Department of Applied Mathematics III, Universitat Politècnica de Catalunya (UPC), 08034 Barcelona, Spain (e-mail: jose.rodellar@upc.es; josep.maria.rossell@upc.es).

Digital Object Identifier 10.1109/TAC.2003.814275 tems with different dimensions, in which solutions of the system with larger dimension include solutions of the system with smaller dimension. The relation between both systems is constructed usually on the base of appropriate linear transformations between the corresponding systems in the original and expanded spaces, where a key role in the selection of appropriate structure of all matrices in the expanded space is played by the so-called complementary matrices [11], [16]. The standard forms of complementary matrices such as aggregations and restrictions have been used in fact as the only well known forms for many years. A contribution to this issue has been presented in [1]-[5] giving a new procedure for a flexible selection of complementary matrices based on appropriate changes of basis in the systems.

When considering control, the following problem arises: give conditions to ensure that a controller designed for the expanded system can be transformed to be implemented in the original system in such a way that the inclusion principle holds for the closed-loop systems. A typical case in the literature is when an original system $\mathbf{S}$ with overlapped components is expanded to a bigger one with a number of disjoint subsystems. Then, decentralized controllers are designed in the expanded system $\tilde{\mathbf{S}}$ and then contracted for implementation in the original system $\mathbf{S}$. This scheme leads to the concept of contractibility.

Early work on contractibility was done for static state controllers in [9], [10], and [14], and for dynamic controllers (including estimators), in [6] and [12], but only with the use of standard complementary matrices in the context of aggregations and restrictions. Contractibility conditions of dynamic controllers were also derived in [7] and [8] for the particular expansion/contraction process referred to as extension, without using complementary matrices. Recently, contractibility of dynamic controllers has been revisited in a more general framework, in which a broader definition of contractibility is proposed to include the specific cases of restrictions, aggregations, and extensions [15], [17].

Suppose given dynamic controllers $\mathbf{C}, \tilde{\mathbf{C}}$ for $\mathbf{S}, \tilde{\mathbf{S}}$. [15, Th. 4] gives contractibility conditions in terms of the parameters of the closed-loop systems $(\mathbf{S}, \mathbf{C})$ and $(\tilde{\mathbf{S}}, \tilde{\mathbf{C}})$ without using complementary matrices. These conditions are general and have a fundamental character, since they do not assume any restriction on the systems and the controllers other than they are linear time-invariant (LTI). However, the conditions involve products and powers of the matrices defining $(\mathbf{S}, \mathbf{C})$ and $(\tilde{\mathbf{S}}$, $\tilde{\mathbf{C}})$. Therefore, there is not a direct way to derive the matrices of $(\tilde{\mathbf{S}}, \tilde{\mathbf{C}})$ for given matrices of $(\mathbf{S}, \mathbf{C})$ or viceversa. This makes the conditions difficult to be directly applied to set up expansion/contraction schemes in practical problems like, for instance, in control design.

This note relies on [15] to give structural properties of contractibility of dynamic controllers in expansion/contraction processes by using complementary matrices. The contractibility condition $\left(\mathrm{D}_{2}\right)$ in [15, Th. 4] is adopted here as the most important case for control design. It is restated in terms of complementary matrices without explicitly involving the matrices defining $(\mathbf{S}, \mathbf{C})$ and $(\tilde{\mathbf{S}}, \tilde{\mathbf{C}})$. This contractibility form results in explicit block structures of complementary matrices. These structures may potentially offer feasible degrees of freedom for specific choices of systemmatricesforbuilding expansion/contractions schemesfor specific problems. A previous work [1] has illustrated this potential in designing overlapping static linear quadratic optimal controllers.

\section{PRoblem Statement}

\section{A. Preliminaries}

Consider the LTI systems

$$
\begin{aligned}
\mathbf{S}: \dot{x} & =A x+B u & \tilde{\mathbf{S}}: \dot{\tilde{x}} & =\tilde{A} \tilde{x}+\tilde{B} \tilde{u} \\
y & =C x & \tilde{y} & =\tilde{C} \tilde{x}
\end{aligned}
$$


where $x(t) \in \mathbb{R}^{\mathbf{n}}, u(t) \in \mathbb{R}^{\mathbf{m}}, y(t) \in \mathbb{R}^{\mathbf{l}}$ are the state, input and output of $\mathbf{S}$ at time $t \in \mathbb{R}^{+}$, and $\tilde{x}(t) \in \mathbb{R}^{\tilde{\mathbf{n}}}, \tilde{u}(t) \in \mathbb{R}^{\tilde{\mathbf{m}}}, \tilde{y}(t) \in \mathbb{R}^{\tilde{\mathbf{l}}}$ are those ones of $\tilde{\mathbf{S}}$. $A, B, C$, and $\tilde{A}, \tilde{B}, \tilde{C}$ are constant matrices of dimensions $n \times n, n \times m, l \times n$ and $\tilde{n} \times \tilde{n}, \tilde{n} \times \tilde{m}, \tilde{l} \times \tilde{n}$, respectively. Suppose that the dimensions of the state, input, and output vectors $x, u, y$ of $\mathbf{S}$ are smaller than (or at most equal to) those of $\tilde{x}, \tilde{u}, \tilde{y}$ of $\tilde{\mathbf{S}}$. Denote $x\left(t ; x_{0}, u\right)$ and $y[x(t)]$ the state trajectory and the corresponding output of $\mathbf{S}$ for a fixed input $u(t)$ and for an initial state $x(0)=x_{0}$, respectively. Similar notations $\tilde{x}\left(t ; \tilde{x}_{0}, \tilde{u}\right)$ and $\tilde{y}[\tilde{x}(t)]$ are used for the state and output trajectories of $\tilde{\mathbf{S}}$.

Let us consider the LTI dynamic controllers

$$
\begin{array}{rlrl}
\mathbf{C}: \dot{z} & =F z+P u+G y & \tilde{\mathbf{C}}: \dot{\tilde{z}}=\tilde{F} \tilde{z}+\tilde{P} \tilde{u}+\tilde{G} \tilde{y} \\
u & =H z+K y+v & \tilde{u} & =\tilde{H} \tilde{z}+\tilde{K} \tilde{y}+\tilde{v}
\end{array}
$$

for the systems $\mathbf{S}$ and $\tilde{\mathbf{S}}$, respectively, where $z(t) \in \mathbb{R}^{\mathbf{p}}$ is the state of $\mathbf{C}$ at time $t \in \mathbb{R}^{+}$and $\tilde{z}(t) \in \mathbb{R}^{\tilde{\mathbf{p}}}$ is this one of $\tilde{\mathbf{C}}$. The vectors $v(t) \in \mathbb{R}^{\mathbf{m}}$, $\tilde{v}(t) \in \mathbb{R}^{\tilde{\mathbf{m}}}$ are new inputs to the corresponding closed-loop systems. The matrices $F, P, G, H, K, \tilde{F}, \tilde{P}, \tilde{G}, \tilde{H}$, and $\tilde{K}$ are constant with appropriate dimensions. Consider the transformations $V: \mathbb{R}^{\mathbf{n}} \longrightarrow \mathbb{R}^{\tilde{\mathbf{n}}}$, $U: \mathbb{R}^{\tilde{\mathbf{n}}} \longrightarrow \mathbb{R}^{\mathbf{n}}, R: \mathbb{R}^{\mathbf{m}} \longrightarrow \mathbb{R}^{\tilde{\mathbf{m}}}, Q: \mathbb{R}^{\tilde{\mathbf{m}}} \longrightarrow \mathbb{R}^{\mathbf{m}}, T: \mathbb{R}^{1} \longrightarrow$ $\mathbb{R}^{\tilde{1}}, S: \mathbb{R}^{\tilde{1}} \longrightarrow \mathbb{R}^{\mathbf{1}}, E: \mathbb{R}^{\mathbf{p}} \longrightarrow \mathbb{R}^{\tilde{\mathbf{p}}}, D: \mathbb{R}^{\tilde{\mathbf{p}}} \longrightarrow \mathbb{R}^{\mathbf{p}}$, where $\operatorname{rank}(V)=n, \operatorname{rank}(R)=m, \operatorname{rank}(T)=l, \operatorname{rank}(E)=p$, and such that $U V=I_{n}, Q R=I_{m}, S T=I_{l}, D E=I_{p}$, where $I_{n}, I_{m}, I_{l}, I_{p}$ are identity matrices of indicated dimensions.

Definition 1: (Inclusion Principle) A system $\tilde{\mathbf{S}}$ is an expansion of the system, $\mathbf{S}$, denoted by $\tilde{\mathbf{S}} \supset \mathbf{S}$, if there exist transformations $(U$, $V, Q, S)$ such that, for any initial state $x_{0}$ of $\mathbf{S}$ and any fixed input $\tilde{u}(t)$ of $\tilde{\mathbf{S}}$, the choice $\tilde{x}_{0}=V x_{0}, u(t)=Q \tilde{u}(t)$ for all $t \geq 0$ implies $x\left(t ; x_{0}, u\right)=U \tilde{x}\left(t ; \tilde{x}_{0}, \tilde{u}\right)$ and $y[x(t)]=S \tilde{y}[\tilde{x}(t)]$, for all $t \geq 0$.

Definition 2: (Contractibility) Suppose $\tilde{\mathbf{S}} \supset \mathbf{S}$. The controller $\tilde{\mathbf{C}}$ for $\tilde{\mathbf{S}}$ is contractible to the controller $\mathbf{C}$ of $\mathbf{S}$, if there exist transformations $(U, V, Q, S, D, E)$ such that, for any initial state $x_{0}$ of $\mathbf{S}$, any initial state $z_{0}$ of $\mathbf{C}$ and any fixed input $\tilde{u}(t)$ of $\tilde{\mathbf{S}}$, the choice $\tilde{z}_{0}=$ $E z_{0}$ implies $z\left(t ; z_{0}, u, y\right)=D \tilde{z}\left(t ; \tilde{z}_{0}, \tilde{u}, \tilde{y}\right)$ and $H z(t)+K y(t)=$ $Q(\tilde{H} \tilde{z}(t)+\tilde{K} \tilde{y}(t))$ for all $t \geq 0$.

Definition 2 states the inclusion $(\tilde{\mathbf{S}}, \tilde{\mathbf{C}}) \supset(\mathbf{S}, \mathbf{C})$ when $\tilde{u}(t)$ is unrestricted in $\tilde{\mathbf{S}}$. This case is important for a control design because it offers the possibility to design completely free a control law for the expanded system $\tilde{\mathbf{S}}$, which is subsequently contracted into the control law for the implementation in the original system $\mathbf{S}$ in such a way that both closed-loop systems satisfy the inclusion relation.

Now, suppose that the pairs of matrices $(U, V),(Q, R),(S, T)$ and $(D, E)$ are given. Then, the matrices $\tilde{A}, \tilde{B}, \tilde{C}, \tilde{F}, \tilde{P}, \tilde{G}, \tilde{H}$ and $\tilde{K}$ can be expressed as

$$
\begin{array}{ll}
\tilde{A}=V A U+M & \tilde{B}=V B Q+N \quad \tilde{C}=T C U+L \\
\tilde{F}=E F D+M_{F} & \tilde{P}=E P Q+Y_{P}, \quad \tilde{G}=E G S+N_{G} \\
\tilde{H}=R H D+L_{H} & \tilde{K}=R K S+J_{K}
\end{array}
$$

where $M, N, L, M_{F}, Y_{P}, N_{G}, L_{H}$, and $J_{K}$ are complementary matrices of appropriate dimensions. The relations between the systems $\mathbf{S}$ and $\tilde{\mathbf{S}}$ in terms of complementary matrices are given in [8], [9], [12]-[14], [16], and [17].

Theorem 1: A system $\tilde{\mathbf{S}}$ is an expansion of the system $\mathbf{S}$ if and only if $U M^{i} V=0, U M^{i-1} N=0, S L M^{i-1} V=0, S L M^{i-1} N=0$ hold for all $i=1, \ldots, \tilde{n}$.

Necessary and sufficient conditions for contractibility by using Definition 2 are given now by the following theorem; see $\left(D_{2}\right)$ in $[15, T h$. 4].
Theorem 2: Suppose $\tilde{\mathbf{S}} \supset \mathbf{S}$. The controller $\tilde{\mathbf{C}}$ for $\tilde{\mathbf{S}}$ is contractible to the controller $\mathbf{C}$ of $\mathbf{S}$ if and only if

$$
\begin{aligned}
& \text { a) } D \tilde{F}^{i} E=F^{i} \\
& \text { b) } D \tilde{F}^{i} \tilde{G} \tilde{C} \tilde{A}^{j} V=F^{i} G C A^{j} \\
& \text { c) } D \tilde{F}^{i} \tilde{G} \tilde{C} \tilde{A}^{j} \tilde{B}=F^{i} G C A^{j} B Q \\
& \text { d) } D \tilde{F}^{i} \tilde{P}=F^{i} P Q \\
& \text { e) } Q \tilde{H} \tilde{F}^{i} E=H F^{i} \\
& \text { f) } Q \tilde{H} \tilde{F}^{i} \tilde{P}=H F^{i} P Q \\
& \text { g) } Q \tilde{H} \tilde{F}^{i} \tilde{G} \tilde{C} \tilde{A}^{j} V=H F^{i} G C A^{j} \\
& \text { h) } Q \tilde{H} \tilde{F}^{i} \tilde{G} \tilde{C} \tilde{A}^{j} \tilde{B}=H F^{i} G C A^{j} B Q \\
& \text { i) } Q \tilde{K} \tilde{C} \tilde{A}^{i} V=K C A^{i} \\
& \text { j) } Q \tilde{K} \tilde{C} \tilde{A}^{i} \tilde{B}=K C A^{i} B Q
\end{aligned}
$$

hold for all $i, j=0,1,2, \ldots$

Remark: Theorem 2 is derived using the contractibility conditions from Definition 2. $z(t)=e^{F t} z_{0}+\int_{0}^{t} e^{F(t-\tau)}[P u(\tau)+G y(\tau)] d \tau$ and $\tilde{z}(t)=e^{\tilde{F} t} \tilde{z}_{0}+\int_{0}^{t} e^{\tilde{F}(t-\tau)}[\tilde{P} \tilde{u}(\tau)+\tilde{G} \tilde{y}(\tau)] d \tau$ are substituted by the terms $y(t)=C\left[e^{A t} x_{0}+\int_{0}^{t} e^{A(t-\sigma)} B u(\sigma) d \sigma\right]$ and $\tilde{y}(t)=$ $\tilde{C}\left[e^{\tilde{A} t} \tilde{x}_{0}+\int_{0}^{t} e^{\tilde{A}(t-\sigma)} \tilde{B} \tilde{u}(\sigma) d \sigma\right]$. The direct comparison of elements between the Taylor series expansions of $e^{F t}, e^{\tilde{F} t}, e^{A t}, e^{\tilde{A} t}$ using the relations in $\tilde{\mathbf{S}}$ and $\mathbf{S}$ results in the assertion.

\section{B. Problem}

The usage of the inclusion principle depends essentially on the choice of the transformation matrices and complementary matrices in the expansion-contraction process [11]. A recent effort has been concentrated on deriving conditions to get generalized structures of complementary matrices for continuous-time LTI systems [1]-[5]. These results include only the contractibility conditions for static state controllers.

The necessary and sufficient contractibility conditions (4) given by Theorem 2 involve products and powers of the matrices $A, B$, and $C$ of system $\mathbf{S}$, the matrices $F, P, G, H$, and $K$ of controller $\mathbf{C}$, as well as the matrices $\tilde{A}, \tilde{B}, \tilde{C}$, and $\tilde{F}, \tilde{P}, \tilde{G}, \tilde{H}$, and $\tilde{K}$ of the expanded system $\tilde{\mathbf{S}}$ and controller $\tilde{\mathbf{C}}$, respectively. Given the matrices of the closed-loop $(\mathbf{S}, \mathbf{C})$, it is difficult to find explicit matrices of $(\tilde{\mathbf{S}}, \tilde{\mathbf{C}})$, and vice versa, such that the conditions (4) are fulfilled. One approach to overcome this problem is by introducing the complementary matrices defined in (3) and expressing the contractibility conditions (4) in terms of these matrices. This new form of the conditions leads to identify block structures of complementary matrices ensuring contractibility, from which the selection of the matrices $\tilde{A}, \tilde{B}, \tilde{C}, \tilde{F}, \tilde{P}, \tilde{G}, \tilde{H}$, and $\tilde{K}$ becomes more feasible with higher degrees of freedom as required in control design. With this approach, the contractibility conditions rely on the appropriate selection of complementary matrices. To the authors knowledge, there is no systematic procedure for the selection of complementary matrices in the case of dynamic controllers.

Therefore, the motivation of this work is to provide a systematic generalization of the structure of complementary matrices for contractibility of dynamic controllers for continuous-time LTI systems to obtain a more flexible tool, mainly for decentralized control design. The problem is formulated as follows.

- To derive necessary and sufficient conditions for contractibility of dynamic controllers for general expansion-contraction transformation matrices given in the form of both unstructured and block structured complementary matrices.

- To derive sufficient conditions for contractibility of dynamic controllers for both general expansion-contraction transformation 
matrices and their particular standard selection given in the form of explicit conditions on blocks of structured complementary matrices.

\section{MAIN RESULTS}

\section{A. Contractibility of Dynamic Controllers}

The following theorem states necessary and sufficient contractibility conditions in terms of the complementary matrices $M, N, L, M_{F}, Y_{P}$, $N_{G}, L_{H}$, and $J_{K}$. In further sections, they will be exploited to identify block structures of these matrices ensuring contractibility.

Theorem 3: Suppose $\tilde{\mathbf{S}} \supset \mathbf{S}$. The controller $\tilde{\mathbf{C}}$ for $\tilde{\mathbf{S}}$ is contractible to the controller $\mathbf{C}$ of $\mathbf{S}$ if and only if

$$
\begin{aligned}
& \text { a) } D M_{F}^{i+1} E=0 \\
& \text { b) } D M_{F}^{i} N_{G}(T C+L V)=0 \quad D M_{F}^{i} N_{G} L M^{j+1} V=0 \\
& \text { c) } D M_{F}^{i} N_{G} L M^{j} N=0 \\
& \text { d) } D M_{F}^{i} Y_{P}=0 \\
& \text { e) } Q L_{H} M_{F}^{i} E=0 \\
& \text { f) } Q L_{H} M_{F}^{i} Y_{P}=0 \\
& \text { g) } Q L_{H} M_{F}^{i} N_{G}(T C+L V)=0 \\
& \quad Q L_{H} M_{F}^{i} N_{G} L M^{j+1} V=0 \\
& \text { h) } Q L_{H} M_{F}^{i} N_{G} L M^{j} N=0 \\
& \text { i) } Q J_{K}(T C+L V)=0 \quad Q J_{K} L M^{i+1} V=0 \\
& \text { j) } Q J_{K} L M^{i} N=0
\end{aligned}
$$

hold for all $i, j=0,1,2, \ldots$

Proof: The proof starts from the expressions (4) that assure the contractibility of a controller. We will prove only the relations $a$ ) and $b$ ) because the remaining conditions follow a similar process. Proof of part $a$ ): Consider the relation $a$ ) given in (4), that is, $D \tilde{F}^{i} E=F^{i}$ together with the relations between the corresponding matrices in $\tilde{\mathbf{S}}$ and $\mathbf{S}$ and the assertion of Theorem 1. We obtain $D E=I_{p}$ for $i=0$ which holds by hypothesis. We get $D\left(E F D+M_{F}\right) E=F$ for $i=1$, that is, $D M_{F} E=0$. In general, $D M_{F}^{i} E=0$ for $i \geq 1$. Then, $D M_{F}^{i+1} E=0$ for all $i \geq 0$. This proves $a$ ). Proof of part $b$ ): Consider the relation $b$ ) given in (4), i.e., $D \tilde{F}^{i} \tilde{G} \tilde{C} \tilde{A}^{j} V=F^{i} G C A^{j}$ for all $i, j=0,1,2, \ldots$ We obtain $D N_{G} L M^{j} V=0$ for $i=0, j \geq 1$ and $D M_{F}^{i} N_{G}(T C+$ $L V)=0$ for $i \geq 0, j=0$ and $D M_{F}^{i} N_{G} L M^{j} V=0$ for $i \geq 1$, $j \geq 1$. Summarizing these relations, $D M_{F}^{i} N_{G}(T C+L V)=0$ and $D M_{F}^{i} N_{G} L M^{j+1} V=0$ for all $i, j \geq 0$.

\section{B. Expansion-Contraction Process of Systems}

Consider the system $\mathbf{S}$ partitioned into $3 \times 3$ blocks such that the diagonal blocks $A_{i i}, B_{i i}$, and $C_{i i}$ have dimensions $n_{i} \times n_{i}, n_{i} \times m_{i}$, $l_{i} \times n_{i}$, respectively, satisfying $n_{1}+n_{2}+n_{3}=n, m_{1}+m_{2}+m_{3}=m$, $l_{1}+l_{2}+l_{3}=l, n_{1}+2 n_{2}+n_{3}=\tilde{n}, m_{1}+2 m_{2}+m_{3}=\tilde{m}$, $l_{1}+2 l_{2}+l_{3}=\tilde{l}$. Suppose subsystems $\mathbf{S}_{\mathbf{1}}$ and $\mathbf{S}_{\mathbf{2}}$ defined by $x_{i}, u_{i}$, (.) $)_{i j}$ for $i, j=1,2$ and $i, j=2,3$, respectively, where $(.)_{i j}$ denotes simultaneously $A_{i j}, B_{i j}$, and $C_{i j}$ in $\mathbf{S}$. Therefore, overlapping appears in $x_{2}, u_{2}$, and $(.)_{22}$. This system overlapping structure defined by these blocks of matrices has been extensively adopted as prototype in the literature. We summarize the necessary most important results about the structure and properties of the complementary matrices such that the inclusion principle is guaranteed.

Suppose given the matrices $V, R$, and $T$. Define $U=\left(V^{t} V\right)^{-1} V^{t}$, $Q=\left(R^{t} R\right)^{-1} R^{t}$, and $S=\left(T^{t} T\right)^{-1} T^{t}$ as the pseudoinverses of $V, R$, and $T$, respectively. Let us consider the change of basis $T_{A}=$ $\left(V W_{A}\right), T_{B}=\left(R W_{B}\right)$, and $T_{C}=\left(T W_{C}\right)$, where $W_{A}, W_{B}$, and $W_{C}$ are chosen such that $\operatorname{Im} W_{A}=\operatorname{Ker} U, \operatorname{Im} W_{B}=\operatorname{Ker} Q$, and
$\operatorname{Im} W_{C}=\operatorname{Ker} S$. The expansion-contraction process between the systems $\mathbf{S}$ and $\tilde{\mathbf{S}}$ with a convenient changes of basis introduced in $\tilde{\mathbf{S}}$ [1], [2], [14], and [16], can be illustrated in the form

$$
\begin{gathered}
\mathbf{S} \rightarrow \tilde{\mathbf{S}} \longrightarrow \tilde{\overline{\mathbf{S}}} \longrightarrow \tilde{\mathbf{S}} \rightarrow \mathbf{S} \\
\mathbb{R}^{\mathrm{n}} \stackrel{V}{\rightarrow} \mathbb{R}^{\tilde{\mathrm{n}}} \stackrel{T_{A}^{-1}}{\longrightarrow} \overline{\mathbb{R}}^{\tilde{\mathrm{n}}} \stackrel{T_{A}}{\longrightarrow} \mathbb{R}^{\tilde{\mathrm{n}}} \stackrel{U}{\rightarrow} \mathbb{R}^{\mathrm{n}} \\
\mathbb{R}^{\mathrm{m}} \stackrel{R}{\rightarrow} \mathbb{R}^{\tilde{\mathrm{m}}} \stackrel{T_{B}^{-1}}{\longrightarrow} \overline{\mathbb{R}}^{\tilde{\mathrm{m}}} \stackrel{T_{B}}{\longrightarrow} \mathbb{R}^{\tilde{\mathrm{m}}} \stackrel{Q}{\rightarrow} \mathbb{R}^{\mathrm{m}} \\
\mathbb{R}^{\mathrm{l}} \stackrel{T}{\rightarrow} \mathbb{R}^{\tilde{1}} \stackrel{T_{C}^{-1}}{\longrightarrow} \overline{\mathbb{R}}^{\tilde{1}} \stackrel{T_{C}}{\longrightarrow} \mathbb{R}^{\tilde{1}} \stackrel{S}{\rightarrow} \mathbb{R}^{\mathrm{l}}
\end{gathered}
$$

where $\tilde{\overline{\mathbf{S}}}$ denotes the expanded system in the new basis.

Denote the relations $\bar{V}=T_{A}^{-1} V, \bar{R}=T_{B}^{-1} R, \bar{T}=T_{C}^{-1} T, \bar{U}=$ $U T_{A}, \bar{Q}=Q T_{B}$, and $\bar{S}=S T_{C}$. Consider the pair of LTI systems

$$
\begin{aligned}
\mathbf{S}: \dot{x} & =A x+B u & \tilde{\overline{\mathbf{S}}}: & \dot{\overline{\bar{x}}}=\tilde{\bar{A}} \tilde{\bar{x}}+\tilde{\bar{B}} \tilde{\bar{u}} \\
y & =C x & \tilde{\bar{y}} & =\tilde{\bar{C}} \tilde{\bar{x}}
\end{aligned}
$$

where the matrices $\tilde{\bar{A}}, \tilde{\bar{B}}$, and $\tilde{\bar{C}}$ have appropriate dimensions. The vectors $\overline{\bar{x}}$, $\tilde{\bar{u}}$, and $\tilde{\bar{y}}$ are defined as $\tilde{\bar{x}}=\bar{V} x, \tilde{\bar{u}}=\bar{R} u$, and $\tilde{\bar{y}}=\bar{T} y$. Denote $\tilde{\bar{A}}=\bar{V} A \bar{U}+\bar{M}, \tilde{\bar{B}}=\bar{V} B \bar{Q}+\bar{N}, \tilde{\bar{C}}=\bar{T} C \bar{U}+\bar{L}$, where new complementary matrices are $\bar{M}=T_{A}^{-1} M T_{A}, \bar{N}=T_{A}^{-1} N T_{B}$, and $\bar{L}=T_{C}^{-1} L T_{A}$. The conditions for the inclusion principle by Theorem 1 are now as follows: $\bar{U} \bar{M}^{i} \bar{V}=0, \bar{U} \bar{M}^{i-1} \bar{N}=0, \bar{S} \bar{L} \bar{M}^{i-1} \bar{V}=0$, and $\bar{S} \bar{L} \bar{M}^{i-1} \bar{N}=0$, for all $i=1,2, \ldots, \tilde{n}$.

Consider appropriately partitioned matrices $M=\left(M_{i j}\right), N=$ $\left(N_{i j}\right), L=\left(L_{i j}\right), i, j=1, \ldots, 4$ in $\tilde{\mathbf{S}}$. Now, denote the matrices $\bar{M}, \bar{N}$ and $\bar{L}$ as follows:

$$
\begin{aligned}
\bar{M} & =\left(\begin{array}{ll}
\bar{M}_{11} & \bar{M}_{12} \\
\bar{M}_{21} & \bar{M}_{22}
\end{array}\right) \\
\bar{N} & =\left(\begin{array}{ll}
\bar{N}_{11} & \bar{N}_{12} \\
\bar{N}_{21} & \bar{N}_{22}
\end{array}\right) \\
\bar{L} & =\left(\begin{array}{ll}
\bar{L}_{11} & \bar{L}_{12} \\
\bar{L}_{21} & \bar{L}_{22}
\end{array}\right)
\end{aligned}
$$

and such that $\bar{M}_{11}, \bar{M}_{22}$ are $n \times n, n_{2} \times n_{2}$ matrices, respectively; $\bar{N}_{11}, \bar{N}_{22}$ are $n \times m, n_{2} \times m_{2}$ matrices, respectively, and $\bar{L}_{11}, \bar{L}_{22}$ are $l \times n, l_{2} \times n_{2}$ matrices, respectively.

The conditions on the blocks $\bar{M}_{i j}, \bar{N}_{i j}$ and $\bar{L}_{i j}, i, j=1,2$ to satisfy the previous conditions for the inclusion principle have been proved in [1], [2]. They are finally reduced to the conditions on submatrices in the form

$$
\begin{aligned}
\bar{M}_{12} \bar{M}_{22}^{i-2} \bar{M}_{21} & =0, \text { for } i=2, \ldots, \tilde{n} \\
\bar{M}_{12} \bar{M}_{22}^{i-2} \bar{N}_{21} & =0 \\
\bar{M}_{12} \bar{M}_{22}^{i-2} \bar{N}_{22} & =0, \text { for } i=2, \ldots, \tilde{n} \\
\bar{L}_{12} \bar{M}_{22}^{i-2} \bar{M}_{21} & =0, \text { for } i=2, \ldots, \tilde{n} \\
\bar{L}_{12} \bar{M}_{22}^{i-2} \bar{N}_{21} & =0 \\
\bar{L}_{12} \bar{M}_{22}^{i-2} \bar{N}_{22} & =0, \text { for } i=2, \ldots, \tilde{n}+1
\end{aligned}
$$

where

$$
\begin{aligned}
\bar{M} & =\left(\begin{array}{cc}
0 & \bar{M}_{12} \\
\bar{M}_{21} & \bar{M}_{22}
\end{array}\right) \\
\bar{N} & =\left(\begin{array}{cc}
0 & 0 \\
\bar{N}_{21} & \bar{N}_{22}
\end{array}\right) \\
\bar{L} & =\left(\begin{array}{cc}
0 & \bar{L}_{12} \\
\bar{L}_{21} & \bar{L}_{22}
\end{array}\right) .
\end{aligned}
$$

\section{Expansion-Contraction Process of Dynamic Controllers}

Analogously to the expansion-contraction of systems $\mathbf{S}$ and $\tilde{\mathbf{S}}$, consider the following scheme for expansion/contraction of controllers $\mathbf{C}$ 
and $\tilde{\mathbf{C}}$ :

$$
\begin{gathered}
\mathbf{C} \rightarrow \tilde{\mathbf{C}} \longrightarrow \tilde{\overline{\mathbf{C}}} \longrightarrow \tilde{\mathbf{C}} \rightarrow \mathbf{C} \\
\mathbb{R}^{\mathbf{p}} \stackrel{\frac{E}{\rightarrow}}{\rightarrow} \mathbb{R}^{\tilde{\mathbf{p}}} \stackrel{T_{F}^{-1}}{\longrightarrow} \overline{\mathbb{R}}^{\tilde{\mathbf{p}}} \stackrel{T_{F}}{\longrightarrow} \mathbb{R}^{\tilde{\mathbf{p}}} \stackrel{D}{\rightarrow} \mathbb{R}^{\mathbf{p}}
\end{gathered}
$$

where $T_{F}=\left(E W_{F}\right)$ and $W_{F}$ satisfies $\operatorname{Im} W_{F}=\operatorname{Ker} D$. Consider the complementary matrices $M_{F}=\left(M_{F_{i j}}\right), Y_{P}=\left(Y_{P_{i j}}\right), N_{G}=$ $\left(N_{G_{i j}}\right), L_{H}=\left(L_{H_{i j}}\right)$, and $J_{K}=\left(J_{K_{i j}}\right)$ for $i, j=1, \ldots, 4$ in $\tilde{\mathbf{C}}$. Consider the block matrices in $\tilde{\overline{\mathbf{C}}}$ as follows:

$$
\begin{aligned}
\bar{M}_{F}=\left(\begin{array}{ll}
\bar{M}_{F_{11}} & \bar{M}_{F_{12}} \\
\bar{M}_{F_{21}} & \bar{M}_{F_{22}}
\end{array}\right) & \bar{Y}_{P}=\left(\begin{array}{ll}
\bar{Y}_{P_{11}} & \bar{Y}_{P_{12}} \\
\bar{Y}_{P_{21}} & \bar{Y}_{P_{22}}
\end{array}\right) \\
\bar{N}_{G}=\left(\begin{array}{ll}
\bar{N}_{G_{11}} & \bar{N}_{G_{12}} \\
\bar{N}_{G_{21}} & \bar{N}_{G_{22}}
\end{array}\right) & \bar{L}_{H}=\left(\begin{array}{ll}
\bar{L}_{H_{11}} & \bar{L}_{H_{12}} \\
\bar{L}_{H_{21}} & \bar{L}_{H_{22}}
\end{array}\right) \\
\bar{J}_{K}=\left(\begin{array}{ll}
\bar{J}_{K_{11}} & \bar{J}_{K_{12}} \\
\bar{J}_{K_{21}} & \bar{J}_{K_{22}}
\end{array}\right) &
\end{aligned}
$$

where $\bar{M}_{F_{11}}, \bar{M}_{F_{22}}$ are $p \times p, p_{2} \times p_{2}$ matrices, respectively. $\bar{Y}_{P_{11}}$ and $\bar{Y}_{P_{22}}$ are $p \times m, p_{2} \times m_{2}$ matrices, respectively. $\bar{N}_{G_{11}}, \bar{N}_{G_{22}}$ are $p \times l$, $p_{2} \times l_{2}$ matrices, respectively. $\bar{L}_{H_{11}}$ and $\bar{L}_{H_{22}}$ are $m \times p, m_{2} \times p_{2}$ matrices, respectively. $\bar{J}_{K_{11}}, \bar{J}_{K_{22}}$ are $m \times l$, and $m_{2} \times l_{2}$ matrices, respectively.

Theorem 4: Suppose $\tilde{\overline{\mathbf{S}}} \supset \mathbf{S}$. The controller $\tilde{\overline{\mathbf{C}}}$ for $\tilde{\overline{\mathbf{S}}}$ is contractible to the controller $\mathbf{C}$ of $\mathbf{S}$ if and only if

$$
\begin{aligned}
& \text { a) } \bar{M}_{F_{11}}=0 \quad \bar{M}_{F_{12}} \bar{M}_{F_{22}}^{i} \bar{M}_{F_{21}}=0 \\
& \text { b) } \bar{N}_{G_{11}} C+\bar{N}_{G_{12}} \bar{L}_{21}=0 \\
& \bar{M}_{F_{12}} \bar{M}_{F_{22}}^{i}\left(\bar{N}_{G_{21}} C+\bar{N}_{G_{22}} \bar{L}_{21}\right)=0 \\
& \left(\bar{N}_{G_{11}} \bar{L}_{12}+\bar{N}_{G_{12}} \bar{L}_{22}\right) \bar{M}_{22}^{j} \bar{M}_{21}=0 \\
& \bar{M}_{F_{12}} \bar{M}_{F_{22}}^{i} \bar{N}_{G_{22}} \bar{L}_{22} \bar{M}_{22}^{j} \bar{M}_{21}=0 \\
& \text { c) } \bar{N}_{G_{12}} \bar{L}_{22} \bar{M}_{22}^{j} \bar{N}_{21}=0 \quad \bar{N}_{G_{12}} \bar{L}_{22} \bar{M}_{22}^{j} \bar{N}_{22}=0 \\
& \bar{M}_{F_{12}} \bar{M}_{F_{22}}^{i} \bar{N}_{G_{22}} \bar{L}_{22} \bar{M}_{22}^{j} \bar{N}_{21}=0 \\
& \bar{M}_{F_{12}} \bar{M}_{F_{22}}^{i} \bar{N}_{G_{22}} \bar{L}_{22} \bar{M}_{22}^{j} \bar{N}_{22}=0 \\
& \text { d) } \bar{Y}_{P_{11}}=0 \quad \bar{Y}_{P_{12}}=0 \quad \bar{M}_{F_{12}} \bar{M}_{F_{22}}^{i} \bar{Y}_{P_{21}}=0 \\
& \bar{M}_{F_{12}} \bar{M}_{F_{22}}^{i} \bar{Y}_{P_{22}}=0 \\
& \text { e) } \bar{L}_{H_{11}}=0 \quad \bar{L}_{H_{12}} \bar{M}_{F_{22}}^{i} \bar{M}_{F_{21}}=0 \\
& \text { f) } \bar{L}_{H_{12}} \bar{M}_{F_{22}}^{i} \bar{Y}_{P_{21}}=0 \quad \bar{L}_{H_{12}} \bar{M}_{F_{22}}^{i} \bar{Y}_{P_{22}}=0 \\
& \text { g) } \bar{L}_{H_{12}} \bar{M}_{F_{22}}^{i}\left(\bar{N}_{G_{21}} C+\bar{N}_{G_{22}} \bar{L}_{21}\right)=0 \\
& \bar{L}_{H_{12}} \bar{M}_{F_{22}}^{i} \bar{N}_{G_{22}} \bar{L}_{22} \bar{M}_{22}^{j} \bar{M}_{21}=0 \\
& \text { h) } \bar{L}_{H_{12}} \bar{M}_{F_{22}}^{i} \bar{N}_{G_{22}} \bar{L}_{22} \bar{M}_{22}^{j} \bar{N}_{21}=0 \\
& \bar{L}_{H_{12}} \bar{M}_{F_{22}}^{i} \bar{N}_{G_{22}} \bar{L}_{22} \bar{M}_{22}^{j} \bar{N}_{22}=0 \\
& \text { i) } \bar{J}_{K_{11}} C+\bar{J}_{K_{12}} \bar{L}_{21}=0 \quad \bar{J}_{K_{12}} \bar{L}_{22} \bar{M}_{22}^{i} \bar{M}_{21}=0 \\
& \text { j) } \bar{J}_{K_{12}} \bar{L}_{22} \bar{M}_{22}^{i} \bar{N}_{21}=0 \quad \bar{J}_{K_{12}} \bar{L}_{22} \bar{M}_{22}^{i} \bar{N}_{22}=0
\end{aligned}
$$

hold for all $i, j \geq 0$, where $\bar{M}_{F}, \bar{Y}_{P}, \bar{N}_{G}, \bar{L}_{H}$, and $\bar{J}_{K}$ have the structure given in (11).

Proof: The proof follows a similar way for all items $a$ ) $-j$ ). Because of this, we prove only the conditions $a$ ) and $b$ ). Proof of part $a$ ): Consider the relation $a$ ) given in (5) in the new basis, that is, $\bar{D} \bar{M}_{F}^{i+1} \bar{E}=0$. We obtain $\bar{M}_{F_{11}}=0$ for $i=0$. We get $\bar{M}_{F_{12}} \bar{M}_{F_{21}}=0$ for $i=1$. In general, $\bar{M}_{F_{12}} \bar{M}_{F_{22}}^{i-1} \bar{M}_{F_{21}}=0$ holds for $i \geq 1$. Then, $\bar{M}_{F_{12}} \bar{M}_{F_{22}}^{i} \bar{M}_{F_{21}}=0$ for all $i \geq 0$. This proves $a$ ). Proof of part $b$ ): Consider the first relation of $b$ ) given in (5) when consider the new basis, $\bar{D} \bar{M}_{F}^{i} \bar{N}_{G}(\bar{T} C+\bar{L} \bar{V})=0$. We obtain $\bar{N}_{G_{11}} C+\bar{N}_{G_{12}} \bar{L}_{21}=0$ for $i=0$. We get $\bar{M}_{F_{12}} \bar{M}_{F_{22}}^{i-1}\left(\bar{N}_{G_{21}} C+\bar{N}_{G_{22}} \bar{L}_{21}\right)=0$, for all $i \geq 1$, that is, $\bar{M}_{F_{12}} \bar{M}_{F_{22}}^{i 2}\left(\bar{N}_{G_{21}} C+\bar{N}_{G_{22}} \bar{L}_{21}\right)=0$ for all $i \geq 0$. Consider $\bar{D} \bar{M}_{F}^{i} \bar{N}_{G} \bar{L} \bar{M}^{j+1} \bar{V}=0$, the second condition of $b$ ) in (5). We get the equations $\left(\bar{N}_{G_{11}} \bar{L}_{12}+\bar{N}_{G_{12}} \bar{L}_{22}\right) \bar{M}_{22}^{j} \bar{M}_{21}=0$ for $i=0, j \geq 0$ and $\bar{M}_{F_{12}} \bar{M}_{F_{22}}^{i-1} \bar{N}_{G_{22}} \bar{L}_{22} \bar{M}_{22}^{j} \bar{M}_{21}=0$ for all $i \geq 1, j \geq 0$. Then,
$\bar{M}_{F_{12}} \bar{M}_{F_{22}}^{i} \bar{N}_{G_{22}} \bar{L}_{22} \bar{M}_{22}^{j} \bar{M}_{21}=0$ holds for all $i, j \geq 0$. This proves part b).

\section{Explicit Sufficient Conditions for Contractibility}

There exist, of course, infinite number of possibilities how to select the complementary matrices satisfying Theorem 4 . The aforementioned conditions on the complementary submatrices are more flexible than the corresponding relations given by Theorem 3 . However, when designing control laws, the designer needs to know explicit conditions assuring the contractibility of the controllers. The particular conditions stated in the following propositions give us packs of sufficient requirements for their submatrices satisfying Theorem 4 .

Proposition 1: Suppose $\tilde{\overline{\mathbf{S}}} \supset \mathbf{S}$. The controller $\tilde{\overline{\mathbf{C}}}$ for $\tilde{\overline{\mathbf{S}}}$ is contractible to the controller $\mathbf{C}$ of $\mathbf{S}$ if $\bar{M}_{F_{11}}=0, \bar{M}_{F_{12}}=0, \bar{Y}_{P_{11}}=0$, $\bar{Y}_{P_{12}}=0, \bar{N}_{G_{11}}=0, \bar{L}_{H_{11}}=0, \bar{L}_{H_{12}}=0, \bar{J}_{K_{11}}=0$, and either

$$
\text { a) } \left.\bar{L}_{21}=0, \bar{L}_{22}=0 \quad \text { or } \quad b\right) \bar{N}_{G_{12}}=0 \quad \bar{J}_{K_{12}}=0
$$

hold, where the matrices $\bar{M}_{F}, \bar{Y}_{P}, \bar{N}_{G}, \bar{L}_{H}, \bar{J}_{K}$ have the structure given in (11).

Proof: The proof is obtained by substituting the above conditions into the relations $a$ ) $-j$ ) given by Theorem 4 .

These conditions are better readable in the block matrix form as follows.

Proposition 2: Suppose $\tilde{\overline{\mathbf{S}}} \supset \mathbf{S}$. The controller $\tilde{\overline{\mathbf{C}}}$ for $\tilde{\overline{\mathbf{S}}}$ is contractible to the controller $\mathbf{C}$ of $\mathbf{S}$ if

$$
\begin{aligned}
\bar{M}_{F} & =\left(\begin{array}{cc}
0 & 0 \\
\bar{M}_{F_{21}} & \bar{M}_{F_{22}}
\end{array}\right) \\
\bar{Y}_{P} & =\left(\begin{array}{cc}
0 & 0 \\
\bar{Y}_{P_{21}} & \bar{Y}_{P_{22}}
\end{array}\right) \\
\bar{L}_{H} & =\left(\begin{array}{cc}
0 & 0 \\
\bar{L}_{H_{21}} & \bar{L}_{H_{22}}
\end{array}\right)
\end{aligned}
$$

and either

$$
\text { a) } \begin{aligned}
\bar{L} & =\left(\begin{array}{cc}
0 & \bar{L}_{12} \\
0 & 0
\end{array}\right) \\
\bar{N}_{G} & =\left(\begin{array}{cc}
0 & \bar{N}_{G_{12}} \\
\bar{N}_{G_{21}} & \bar{N}_{G_{22}}
\end{array}\right) \\
\bar{J}_{K} & =\left(\begin{array}{cc}
0 & \bar{J}_{K_{12}} \\
\bar{J}_{K_{21}} & \bar{J}_{K_{22}}
\end{array}\right)
\end{aligned}
$$

or

$$
\text { b) } \begin{aligned}
\bar{N}_{G} & =\left(\begin{array}{cc}
0 & 0 \\
\bar{N}_{G_{21}} & \bar{N}_{G_{22}}
\end{array}\right) \\
\bar{J}_{K} & =\left(\begin{array}{cc}
0 & 0 \\
\bar{J}_{K_{21}} & \bar{J}_{K_{22}}
\end{array}\right)
\end{aligned}
$$

hold, where the matrices $\bar{M}_{F}, \bar{Y}_{P}, \bar{N}_{G}, \bar{L}_{H}$, and $\bar{J}_{K}$ have the structure given in (11).

Proof: The proof is straightforward when using Proposition 1.

\section{E. Particular Selection of Complementary Matrices}

Consider the overlapping structure defined above as a prototype case. Let us use the following transformation matrices $V, R, T$, and $E$ :

$$
\begin{aligned}
V & =\left(\begin{array}{ccc}
I_{n_{1}} & 0 & 0 \\
0 & I_{n_{2}} & 0 \\
0 & I_{n_{2}} & 0 \\
0 & 0 & I_{n_{3}}
\end{array}\right) \quad R=\left(\begin{array}{ccc}
I_{m_{1}} & 0 & 0 \\
0 & I_{m_{2}} & 0 \\
0 & I_{m_{2}} & 0 \\
0 & 0 & I_{m_{3}}
\end{array}\right) \\
T & =\left(\begin{array}{ccc}
I_{l_{1}} & 0 & 0 \\
0 & I_{l_{2}} & 0 \\
0 & I_{l_{2}} & 0 \\
0 & 0 & I_{l_{3}}
\end{array}\right) \quad E=\left(\begin{array}{ccc}
I_{p_{1}} & 0 & 0 \\
0 & I_{p_{2}} & 0 \\
0 & I_{p_{2}} & 0 \\
0 & 0 & I_{p_{3}}
\end{array}\right) .
\end{aligned}
$$


The changes of basis (6) for $\tilde{\mathbf{S}}$ and (10) for $\tilde{\mathbf{C}}$ are given by

$$
\begin{aligned}
T_{A} & =\left(\begin{array}{cccc}
I_{n_{1}} & 0 & 0 & 0 \\
0 & I_{n_{2}} & 0 & I_{n_{2}} \\
0 & I_{n_{2}} & 0 & -I_{n_{2}} \\
0 & 0 & I_{n_{3}} & 0
\end{array}\right) \\
T_{A}^{-1} & =\left(\begin{array}{cccc}
I_{n_{1}} & 0 & 0 & 0 \\
0 & \frac{1}{2} I_{n_{2}} & \frac{1}{2} I_{n_{2}} & 0 \\
0 & 0 & 0 & I_{n_{3}} \\
0 & \frac{1}{2} I_{n_{2}} & -\frac{1}{2} I_{n_{2}} & 0
\end{array}\right)
\end{aligned}
$$

where $I_{n_{i}}$ denote the identity matrices of orders $n_{i}, i=1,2,3$. Analogously for $T_{B}, T_{B}^{-1}, T_{C}, T_{C}^{-1}$, and $T_{F}, T_{F}^{-1}$ in schemes (6) and (10), respectively. The matrices $M$ and $N$ in $\mathbf{S}$ have the form

$$
M=\left(\begin{array}{cccc}
0 & M_{12} & -M_{12} & 0 \\
M_{21} & M_{22} & M_{23} & M_{24} \\
-M_{21} & -\left(M_{22}+M_{23}+M_{33}\right) & M_{33} & -M_{24} \\
0 & M_{42} & -M_{42} & 0
\end{array}\right)
$$

and

$$
N=\left(\begin{array}{cccc}
0 & 0 & 0 & 0 \\
N_{21} & N_{22} & N_{23} & N_{24} \\
-N_{21} & -N_{22} & -N_{23} & -N_{24} \\
0 & 0 & 0 & 0
\end{array}\right)
$$

The matrix $L$ has the same structure as $M$. The propositions derived in the previous subsection has the following form in $\mathbf{S}$ when selecting the transformations given in (15).

Proposition 3: Suppose $\tilde{\mathbf{S}} \supset \mathbf{S}$. The controller $\tilde{\mathbf{C}}$ for $\tilde{\mathbf{S}}$ is contractible to the controller $\mathbf{C}$ for $\mathbf{S}$ if

$$
\begin{aligned}
M_{F} & =\left(\begin{array}{cccc}
0 & 0 & 0 & 0 \\
M_{F_{21}} & M_{F_{22}} & M_{F_{23}} & M_{F_{24}} \\
-M_{F_{21}} & -M_{F_{22}} & -M_{F_{23}} & -M_{F_{24}} \\
0 & 0 & 0 & 0
\end{array}\right) \\
Y_{P} & =\left(\begin{array}{cccc}
0 & 0 & 0 & 0 \\
Y_{P_{21}} & Y_{P_{22}} & Y_{P_{23}} & Y_{P_{24}} \\
-Y_{P_{21}} & -Y_{P_{22}} & -Y_{P_{23}} & -Y_{P_{24}} \\
0 & 0 & 0 & 0
\end{array}\right) \\
L_{H} & =\left(\begin{array}{cccc}
0 & 0 & 0 & 0 \\
L_{H_{21}} & L_{H_{22}} & L_{H_{23}} & L_{H_{24}} \\
-L_{H_{21}} & -L_{H_{22}} & -L_{H_{23}} & -L_{H_{24}} \\
0 & 0 & 0 & 0
\end{array}\right)
\end{aligned}
$$

and either

$$
\begin{aligned}
& \text { a) } L=\left(\begin{array}{llll}
0 & L_{12} & -L_{12} & 0 \\
0 & L_{22} & -L_{22} & 0 \\
0 & L_{22} & -L_{22} & 0 \\
0 & L_{42} & -L_{42} & 0
\end{array}\right) \\
& N_{G}=\left(\begin{array}{cccc}
0 & N_{G_{12}} & -N_{G_{12}} & 0 \\
N_{G_{21}} & N_{G_{22}} & N_{G_{23}} & N_{G_{24}} \\
-N_{G_{21}} & -\left(N_{G_{22}}+N_{G_{23}}+N_{G_{33}}\right) & N_{G_{33}} & -N_{G_{24}} \\
0 & N_{G_{42}} & -N_{G_{42}} & 0
\end{array}\right) \\
& J_{K}=\left(\begin{array}{cccc}
0 & J_{K_{12}} & -J_{K_{12}} & 0 \\
J_{K_{21}} & J_{K_{22}} & J_{K_{23}} & J_{K_{24}} \\
-J_{K_{21}} & -\left(J_{K_{22}}+J_{K_{23}}+J_{K_{33}}\right) & J_{K_{33}} & -J_{K_{24}} \\
0 & J_{K_{42}} & -J_{K_{42}} & 0
\end{array}\right)
\end{aligned}
$$

or

$$
\text { b) } \begin{aligned}
N_{G} & =\left(\begin{array}{cccc}
0 & 0 & 0 & 0 \\
N_{G_{21}} & N_{G_{22}} & N_{G_{23}} & N_{G_{24}} \\
-N_{G_{21}} & -N_{G_{22}} & -N_{G_{23}} & -N_{G_{24}} \\
0 & 0 & 0 & 0
\end{array}\right) \\
J_{K} & =\left(\begin{array}{cccc}
0 & 0 & 0 & 0 \\
J_{K_{21}} & J_{K_{22}} & J_{K_{23}} & J_{K_{24}} \\
-J_{K_{21}} & -J_{K_{22}} & -J_{K_{23}} & -J_{K_{24}} \\
0 & 0 & 0 & 0
\end{array}\right)
\end{aligned}
$$

holds.

Proof: The proof is straightforward when using Proposition 2.

\section{CONCLUSION}

The main result contributed by this note is a set of contractibility conditions for dynamic controllers for LTI systems in terms of the complementary matrices involved in the expansion/contraction framework of the inclusion principle. For general expansion/contraction transformations, necessary and sufficient conditions for contractibility are proved. The conditions involve unstructured as well as block structured complementary matrices. The block structure offers a higher degree of freedom in selection of complementary matrices as compared with previous well known results. This structure is exploited to obtain explicit sufficient requirements for blocks of complementary matrices ensuring contractibility. It is useful for enabling flexible choices of such matrices. Specific choices are finally given for a particular class of expansion/contraction transformation matrices.

\section{REFERENCES}

[1] L. Bakule, J. Rodellar, and J. M. Rossell, "Generalized selection of complementary matrices in the inclusion principle," IEEE Trans. Automat. Contr., vol. 45, pp. 1237-1243, June 2000.

[2] _ - "Structure of expansion-contraction matrices in the inclusion principle for dynamic systems," SIAM J. Matrix Anal. Applicat., vol. 21, no. 4, pp. 1136-1155, 2000.

[3] - "Controllability-observability of expanded composite systems," Linear Alg. Applicat., vol. 332-334, pp. 381-400, 2001.

[4] - "Overlappig quadratic optimal control of linear time-varying commutative systems," SIAM J. Control Optim., vol. 40, no. 5, pp. 1611-1627, 2002.

[5] L. Bakule, J. Rodellar, J. M. Rossell, and P. Rubió, "Preservation of controllability-observability in expanded systems," IEEE Trans. Automat. Contr., vol. 46, pp. 1155-1162, July 2001.

[6] M. Hodžić and D. D. Šiljak, "Decentralized estimation and control with overlapping information sets," IEEE Trans. Automat. Contr., vol. AC-31, pp. 83-86, Jan. 1986.

[7] A. İftar, "Decentralized estimation and control with overlapping input, state, and output decomposition," Automatica, vol. 29, no. 2, pp. 511-516, 1993

[8] - "Overlapping decentralized dynamic optimal control," Int. J. Control, vol. 58, no. 1, pp. 187-209, 1993.

[9] A. İftar and Ü. Özgüner, "Contractible controller design and optimal control with state and input inclusion," Automatica, vol. 26, no. 3, pp. 593-597, 1990.

[10] M. Ikeda and D. D. Šiljak, "Overlapping decompositions, expansions and contractions of dynamic systems," Large Scale Syst., vol. 1, no. 1, pp. 29-38, 1980.

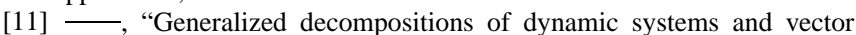
lyapunov functions," IEEE Trans. Automat. Contr., vol. AC-26, pp. 1118-1125, Oct. 1981

[12] —, "Overlapping decentralized control with input state, and output inclusion," Control Theory Adv. Technol., vol. 2, no. 2, pp. 155-172, 1986.

[13] M. Ikeda, D. D. Šiljak, and D. E. White, "Decentralized control with overlapping information sets," J. Optim. Theory Applicat., vol. 34, no. 2, pp. 279-310, 1981.

[14] _ , "An inclusion principle for dynamic systems," IEEE Trans. Automat. Contr., vol. AC-29, pp. 244-249, Mar. 1984. 
[15] S. S. Stanković and D. D. Šiljak, "Contractibility of overlapping decentralized control," Syst. Control Lett., vol. 44, pp. 189-200, 2001.

[16] D. D. Šiljak, Decentralized Control of Complex Systems. New York: Academic, 1991.

[17] D. D. Šiljak, S. M. Mladenović, and S. S. Stanković, "Overlapping decentralized observation and control of a platoon of vehicles," in Proc. Amer. Control Conf., San Diego, CA, 1999, pp. 4522-4526.

\section{Semiglobal Stabilization and Output Regulation of Singular Linear Systems With Input Saturation}

Weiyao Lan and Jie Huang

\begin{abstract}
The semiglobal stabilization problem and output regulation problem of singular linear systems subject to input saturation are addressed. A reduced-order normal system is obtained by a standard coordinate transformation. It is further shown that the controller that solves the stabilization (output regulation) problem of the reduced-order normal systems also solves the stabilization (output regulation) problem of the original singular systems.
\end{abstract}

Index Terms-Input saturation, output regulation, singular system, stabilization.

\section{INTRODUCTION}

Singular systems arise in a variety of practical systems such as networks, circuits, power systems, and so on [10]. Since the late 1970s, there has been extensive study on singular systems. However, singular systems subject to input saturation are hardly studied. In this note, we will consider two important control problems for singular systems subject to input saturation, namely, the output feedback semiglobal stabilization problem and the output feedback semiglobal output regulation problem. For normal linear systems with saturation actuators, the semiglobal stabilization problem and the semiglobal output regulation problem have been thoroughly studied in quite a few papers ([12]-[14]) and two monographs [15] and [16]. In particular, it was shown in [12] that one can semiglobally stabilize a linear system subject to input saturation using linear feedback control laws if the system is asymptotically null controllable with bounded controls. Also, solvability conditions for the output regulation problem of linear systems subject to input saturation was established in [14].

In this note, under some standard assumptions, by employing the familiar system equivalence technique, we will convert a singular system into a reduced-order normal system. Further, we will show that the reduced-order normal systems satisfy the solvability conditions of the semiglobal stabilization (output regulation) problem. Thus, a normal output feedback controller can be constructed explicitly to solve the semiglobal stabilization (output regulation) problem for the reducedorder normal systems with input saturation. Finally, we will show that

Manuscript received October 30, 2002; revised March 4, 2003. Recommended by Associate Editor Z. Lin. This work was supported in part by the Hong Kong Research Grant Council under Grant CUHK 4316/02E.

W. Lan is with the Department of Automation and Computer-Aided Engineering, The Chinese University of Hong Kong, Hong Kong.

J. Huang is with the Department of Automation and Computer-Aided Engineering, The Chinese University of Hong Kong, Hong Kong. He is also with the School of Automation Science and Engineering, South China University of Technology (e-mail: jhuang@ acae.cuhk.edu.hk).

Digital Object Identifier 10.1109/TAC.2003.814276 the same controller also solves the semiglobal stabilization (output regulation) problem for the original singular systems with input saturation.

It should be noted that the output regulation problem for linear singular systems without input saturation is studied in [2] and [11]. The output regulation problem for singular nonlinear systems is formulated and solved in [8]. However, technically, this note is more relevant to [12]-[16].

Notations: For an $n$-dimensional vector $x=\left(x_{1}, x_{2}, \ldots, x_{n}\right)^{T}$, $\|x\| \triangleq\left(\left|x_{1}\right|^{2}+\left|x_{2}\right|^{2}+\cdots+\mid x_{n} \|^{2}\right)^{1 / 2}$, and $|x|_{\infty} \triangleq{ }_{i}{ }^{\max }\left\{\left|x_{i}\right|\right\}$. For a piecewise continuous bounded function $v:[0, \infty) \rightarrow \Re^{m},\|v\|_{\infty} \triangleq$ $\sup _{t>0}|v(t)|_{\infty}$, and for $T \geq 0,\|v\|_{\infty, T} \triangleq \sup _{t>T}|v(t)|_{\infty}$.

This note is organized as follows. In Section II, we formulate the stabilization problem and output regulation problem of the singular linear systems with input saturation. Section III solves the stabilization problem for the singular systems with input saturation, while the output regulation problem is considered in Section IV. An example for semiglobal output regulation problem is given in Section V. Finally, Section VI concludes this note.

\section{Problem Statement and Preliminaries}

A singular linear system subject to input saturation is described as follows:

$$
\begin{aligned}
E \dot{x} & =A x+B \sigma(u) \\
y & =C x
\end{aligned}
$$

where $x \in \Re^{n}$ is the system state, $u \in \Re^{m}$ the control input, and $y \in \Re^{p}$ the measurable output. $E, A \in \Re^{n \times n}, B \in \Re^{n \times m}$, and $C \in$ $\Re^{p \times n}$ are constant matrices. Without loss of generality, we assume that $E$ is singular and $(E, A)$ is regular, i.e, $\operatorname{det}(s E-A) \equiv 0 . \sigma(\cdot)$ is a vector-valued saturation function defined as

$$
\sigma(s)=\left[\bar{\sigma}\left(s_{1}\right), \bar{\sigma}\left(s_{2}\right), \cdots, \bar{\sigma}\left(s_{m}\right)\right]^{T}
$$

where

$$
\bar{\sigma}\left(s_{i}\right)=\left\{\begin{array}{cl}
s_{i}, & \text { if }\left|s_{i}\right| \leq 1 \\
-1, & \text { if } s_{i}<-1 \\
1, & \text { if } s_{i}>1 .
\end{array}\right.
$$

We will consider the normal output feedback control law of the form

$$
\begin{aligned}
& u=K z+J y \\
& \dot{z}=F z+G y+H \sigma(u)
\end{aligned}
$$

where $z \in \Re^{n_{z}}$ for some integer $n_{z}>0, F \in \Re^{n_{z} \times n_{z}}, G \in \Re^{n_{z} \times p}$, $H \in \Re^{n_{z} \times m}, K \in \Re^{m \times n_{z}}$, and $J \in \Re^{m \times p}$ are constant matrices to be determined.

\section{A. Semiglobal Stabilization Problem}

Given any compact sets $X_{0} \subset \Re^{n}$ containing the origin and $Z_{0} \subset$ $\Re^{n} z$ containing the origin, find an output feedback control law (3) such that for all $(x(0), z(0)) \in X_{0} \times Z_{0}$, the solution of the closed-loop system consisting of (1) and (3)

$$
\begin{aligned}
E \dot{x} & =A x+B \sigma(K z+J C x) \\
\dot{z} & =F z+G C x+H \sigma(K z+J C x)
\end{aligned}
$$

exists for all $t \geq 0$ and there exist $\alpha>0$ and $\beta>0$ such that

$$
\left\|\begin{array}{l}
x(t) \\
z(t)
\end{array}\right\| \leq \alpha e^{-\beta t}\left\|\begin{array}{l}
x(0) \\
z(0)
\end{array}\right\|, \quad t>0 .
$$

